\title{
Frühzeitige Insulinisierung bringt Vorteile
}

- Dass sich eine strenge Einstellung des Blutzuckers für Typ-2-Diabetiker auch in einer Reduktion makrovaskulärer Endpunkte auszahlt, bestätigen zwei aktuelle Metaanalysen. Dies trifft jedoch nur zu, wenn der Diabetes frühzeitig gut eingestellt wird. Dazu gehört auch, den Beginn der Insulintherapie nicht zu lange hinauszuzögern.

Lässt man den Diabetes erst über viele Jahre "vergammeln", wird die zu späte schärfere Einstellung zu Übersterblichkeit führen, warnte Prof. Reinhard Bretzel, Gießen. Zum Zeitpunkt der Diagnose eines Typ-2-Diabetes ist die Betazellfunktion bereits auf $40-50 \%$ reduziert und nimmt danach jedes Jahr um weitere $4-6 \%$ ab. Entsprechend muss die antidiabetische Therapie stufenweise intensiviert werden, d.h. dass auch rechtzeitig Insulin integriert werden muss. Dass "rechtzeitig " "möglichst früh" bedeutet, legen Studien nahe, in denen eine frühe Insulinisierung die residuale Betazellfunktion stabilisieren, teilweise sogar bessern konnte.

Auch in der aktuellen DDG-Leitlinie gab es ein "Upgrade" für die Insulintherapie: Wenn bereits die initiale Therapie mit Metformin versagt hat $\left(\mathrm{HbA}_{1 \mathrm{c}}-\right.$ Wert $\left.\geq 7,5 \%\right)$, kann mit Insulin kombiniert werden. In ei- ner aktuellen Metaanalyse von Studien mit mindestens 24 Wochen Dauer untersuchten de Vries et al., was die Einstellung auf Insulin glargin und Metformin bei Typ2-Diabetikern bringt: Der $\mathrm{HbA}_{1 \mathrm{c}}$-Wert nahm von $8,7 \%$ auf $7 \%$ ab, der Nüchternblutzucker von 182 auf $108 \mathrm{mg} / \mathrm{dl}$, bei geringer Hypoglykämierate.

\section{Zeitgemäß Blutzucker messen}

Für die Blutzuckermessung bietet SanofiAventis moderne Geräte wie den iBGStar ${ }^{\oplus}$. Wie der Profi-Sprinter und Typ-1-Diabetiker Daniel Schnelting, Rhede, deutlich machte, ist dieses Gerät zu seinem ständigen Begleiter geworden. Es kann kabellos über den Apple-Dock-Connector direkt an das iPhone angeschlossen werden, sodass die Messwerte auf dem Touchscreen angezeigt werden. Die Werte können auf dem iPhone verwaltet, heruntergeladen und direkt dem Arzt übermittelt werden. Das Gerät verschafft dem Diabetiker maximale Unabhängigkeit.

- Dr. Angelika Bischoff

Quelle: Pressekonferenz „Im Fokus des modernen Diabetesmanagements: Blutzuckerkontrolle und Therapieoptionen", DDG-Jahrestagung, Stuttgart, Mai 2012 (Veranstalter: Sanofi Aventis)
Kurz notiert

Diabetesaktion beim RheinRadeln

- Das RheinRadeln war erstmals Anlaufstelle für die bundesweite Aktion „Wissen was bei Diabetes zählt: Gesünder unter 7", die die Firma Sanofi seit 2005 mehrmals jährlich gemeinsam mit inzwischen 24 Kooperationspartnern wie Fachgesellschaften, Patientenorganisationen und Krankenkassen durchführt. Über $800 \mathrm{Be}$ sucher nutzten die Gelegenheit, sich einem Diabetes-Risikocheck zu unterziehen. Auch 22 Diabetiker waren dabei, um unter dem Motto "messen und radeln" zu demonstrieren, dass sich Bewegung unmittelbar auf die Blutzuckerwerte auswirkt.

Dass der $\mathrm{HbA}_{1 \mathrm{c}}$-Idealwert häufig überschritten wird, zeigt die Auswertung der fast 2600 RisikocheckBögen, die bei Diabetes-Aktionen 2011 ausgefüllt wurden. Nahezu jeder vierte Teilnehmer war diagnostizierter Diabetiker, drei Viertel von ihnen ließen den $\mathrm{HbA}_{1 \mathrm{c}}$-Wert bestimmen: $43 \%$ waren mit einem Wert über 7 schlecht eingestellt.

- Ingeborg Brödlein

Quelle: Aktion „Wissen was bei Diabetes zählt: Gesünder unter 7", Mai 2012 (Veranstalter: Sanofi-Aventis)

\section{Großstudie mit Edoxaban}

\section{ENGAGE-AF testet individualisierte Antikoagulation}

- Bei der klinischen Erprobung des direkten Faktor-Xa-Inhibitors Edoxaban in der Schlaganfallprophylaxe bei Vorhofflimmern setzen die Studienautoren auf einen individuellen Therapieansatz: „Wir wissen, dass das Blutungsrisiko dieser Patienten in Abhängigkeit von Alter, Gewicht und Nierenfunktion variiert und nach Ablation generell etwas höher ausfällt", erklärte Prof. Robert Giugliano, Boston. Deshalb wird Edoxaban in der ENGAGE-AF-TIMI-48-Studie in zwei unterschiedlichen Dosierungen - $30 \mathrm{mg} / \mathrm{d}$ und $60 \mathrm{mg} / \mathrm{d}$ - untersucht. Das Design erlaubt weitere Dosisanpassungen im Laufe der Studie. „Auf diese Weise können wir dem Patienten eine besonders sichere Therapie anbieten", sagte Giugliano.

\section{Klinische Studie mit der bisher größten Patientenzahl}

ENGAGE-AF-TIMI 48 ist die klinische Studie bei Vorhofflimmern mit der bisher größten Patientenzahl ( $\mathrm{n}=21$ 105), dem längsten Follow-up (über zwei Jahre) und der zu erwartenden größten Anzahl von Endpunktereignissen. Es handelt sich um eine Doppelblindstudie von höchster Qualität, so Giuliano. Über $50 \%$ der Patienten weisen mit einem $\mathrm{CHADS}_{2}$-VASC-Score von 3 oder größer ein hohes Schlaganfallrisiko auf. Die Autoren bemühen sich um eine besonders gut eingestellte Vitamin-K-AntagonistenTherapie in der Kontrollgruppe. Dennoch sind sie zuversichtlich, eine überlegene Sicherheit der Edoxaban-Therapie aufzeigen zu können. Die Ergebnisse der Studie werden Ende 2013 erwartet.

- Dr. med. Dirk Einecke

Quelle: Pressekonferenz „Edoxaban, an investigational oral anticoagulant", ESC-Kongress, München, August 2012 (Veranstalter: Daiichi-Sankyo) 